Canadian

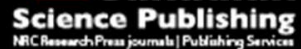

Canadian Journal of Physiology and Pharmacology Revue canadienne de physiologie et pharmacologie

\title{
Effects of estradiol on measurements of conduit artery endothelial function after ischemia and reperfusion in premenopausal women
}

\begin{tabular}{|r|l|}
\hline Journal: & Canadian Journal of Physiology and Pharmacology \\
\hline Manuscript ID & cjpp-2015-0589.R1 \\
\hline Manuscript Type: & Article \\
\hline Complete List of Authors: & $\begin{array}{l}\text { Luca, Mary Clare; Mount Sinai Hospital } \\
\text { Liuni, Andrew; Mount Sinai Hospital } \\
\text { Harvey, Paula; Women \'s College Hospital, Cardiology } \\
\text { Mak, Susanna; Mount Sinai Hospital } \\
\text { Parker, John; Mount Sinai Hospital, Cardiology }\end{array}$ \\
\hline Keyword: & estrogen, endothelium, ischemia reperfusion \\
\hline
\end{tabular}


Effects of estradiol on measurements of conduit artery endothelial function after ischemia and reperfusion in premenopausal women

Running Title: Estradiol and human ischemia reperfusion injury

Mary Clare Luca BSc, Andrew Liuni BSc, Paula Harvey MD, Susanna Mak MD PhD, John D Parker MD

From the Division of Cardiology, Mount Sinai and University Health Network Hospitals, University of Toronto, Canada (M.L., A.L., S.M., J.D.P.); Department of Pharmacology, University of Toronto, Canada (M.L., A.L., J.D.P.); Division of Cardiology, Department of Medicine, Women's College Hospital, University of Toronto, Canada (P.H.)

Total Word count: 4195 (including title page, abstract, text, references, tables and figure legends)

For correspondence

John D Parker MD

Division of Cardiology, Mount Sinai and University Health Network Hospitals

600 University Avenue, Suite 1609

Toronto, Canada, M5G 1 X5

Phone: 1-416-586-4794

Fax: 1-416-586-8413

Email: john.parker@uhn.on.ca 


\begin{abstract}
In premenopausal women ovarian steroids are felt to play a role in the prevention of cardiovascular disease. We aimed to assess whether menstrual cycle variations in estrogen can modify the response to ischemia/reperfusion (IR) injury in humans. In an investigator-blind crossover study in 10 healthy, pre-menopausal women with regular menstrual cycles. They had flow-mediated dilatation (FMD) measured by ultrasound in the radial artery before and after IR (15 minutes of brachial artery ischemia, 15 minutes of reperfusion) during both the early and late follicular phases of the menstrual cycle. The order of these visits was not randomized. IR significantly blunted FMD in the early follicular phase (pre-IR: $7.1 \pm 1.0 \%$; post-IR: $3.6 \pm 1.0 \%$, $\mathrm{P}=0.01)$ when estradiol levels were low $(148.4 \pm 19.8 \mathrm{pmol} / \mathrm{L})$. Conversely, FMD was preserved after IR during the late follicular phase (pre-IR: $7.2 \pm 0.9 \%$; post-IR: $7.0 \pm 0.8 \%, \mathrm{P}=\mathrm{NS}, \mathrm{P}=0.03$ compared to early follicular) when estradiol levels were high $(825.7 \pm 85.8 \mathrm{pmol} / \mathrm{L}, \mathrm{P}<0.001$ compared to early follicular). There was a significant inverse relationship between estradiol concentration and IR-induced endothelial dysfunction (i.e. change in FMD after IR) $(r=0.59$, $\mathrm{r} 2=0.36, \mathrm{P}<0.01)$. These findings demonstrate, for the first time in humans, a clear relationship between the cyclical changes in serum concentrations of estradiol and the endothelium's response to IR.
\end{abstract}

Keywords Endothelium; ischemia; reperfusion; estradiol; early follicular phase, late follicular phase, flow-mediated dilation 


\section{Introduction}

A fundamental paradox in the management of low-flow ischemia is the fact that reperfusion, while critical to survival, is itself a major cause of damage to the myocardium, vasculature and other tissues; a phenomenon known as ischemia and reperfusion (IR) injury. Endothelial cells are very susceptible to, and actively participate in the process of IR injury since dysfunction and/or damage to the endothelial layer of the macro- and microcirculation can impair effective reperfusion of ischemic tissue (Abela and Homer-Vanniasinkham 2003; Kharbanda et al. 2001). This state of "endothelial dysfunction" can exacerbate tissue damage caused by IR through induction of vasoconstriction and the activation of neutrophils and platelets, all leading to further impairment in blood flow (Kharbanda et al. 2001). Thus, the endothelium is very sensitive to IR injury, and at the same time is a major determinant of the capacity of a tissue to recover from IR. As a result, studies aimed at protecting the endothelium from IR are of considerable clinical interest.

Importantly, it has been demonstrated that certain pharmacologic agents are able to provide protection in the setting of IR, a phenomenon referred to as pharmacologic preconditioning. For example, protective effects of delta opioids (through the recruitment of cyclooxygenase-2 (COX2)) (Bolli 2000), angiotensin converting enzyme inhibitors as well as angiotensin receptor blockers (Zhu et al. 2000) have been demonstrated in animal models of IR. In humans, agents such as sildenafil (Gori et al. 2005), organic nitrates (Dragoni et al. 2007), and rosuvastatin (Liuni et al. 2010) have been shown to prevent IR-induced damage to the endothelium in the peripheral vasculature. 
The vast majority of women who develop cardiovascular disease are postmenopausal. This fact suggests a role for estradiol as a modifier of the natural history of cardiovascular disease (Dubey et al. 2005). Animal models of IR have demonstrated the ability of estrogens to decrease infarct size, preserve cardiac and endothelial function, as well as to reduce the bioavailability of reactive oxygen species and markers of inflammation (Murphy and Steenbergen 2007). In humans, estradiol is known to have several protective cardiovascular effects, including the capacity to reduce blood pressure, inhibit smooth muscle cell proliferation, lower cholesterol, and augment endothelium-dependent vascular responses (Dubey et al. 2005). The effect of estradiol on vascular function following IR injury in humans is unknown. Therefore, we aimed to determine whether the peak in estradiol which occurs during the late follicular (LF) phase of the menstrual cycle would prevent IR induced endothelial dysfunction in the forearm vasculature in humans as compared to the effect of IR during the low estrogen, early follicular (EF) phase.

\section{Methods}

The study was approved by the Mount Sinai Research Ethics Board and all subjects gave written informed consent. Studies were conducted in a quiet, temperature- and humidity-controlled environment.

\section{Population}

Ten healthy, pre-menopausal female volunteers with regular menstrual cycles (18-29 years old) were enrolled in this investigator-blind, randomized, crossover trial. All subjects were required to fast and abstain from caffeine for 14 hours prior to the study. Exclusion criteria included any active disease, the use of medications (including supplemental vitamins), the use of contraceptive 
medication in the last six months, current or previous pregnancy, as well as risk factors for cardiovascular disease such as hypertension, smoking, hypercholesterolemia and a family history of premature coronary artery disease. None of the participants had ever been diagnosed with polycystic ovarian syndrome. Table 1 summarizes the characteristics of the study participants.

\section{Monitoring Phase}

Study participants were given a menstrual cycle log where they recorded the timing and length of their menstrual cycles for two complete cycles. Subjects used the Clear Blue digital ovulation kit (McKesson Canada) each month to document the surge in luteinizing hormone (LH) in the urine that occurs just prior to ovulation in order to predict the abrupt rise in estrogen in the subsequent cycle. The reliability of this technique to detect ovulation has previously been established (Guermandi et al. 2001). Study participants were studied at two phases of the menstrual cycle: the EF phase (days 1-4 after the onset of menstruation) and the LF phase (days 10-13 days after the onset of menstruation). Participants had the first study visit performed in either the EF (8 subjects) or the LF (2 subjects) phase in an investigator-blind manner. Visits were performed according to participant availability and the phase of the cycle in which each participant had their first visit was not randomized. The average time between the two study visits was 25 days.

\section{Study Protocol}

After 10 minutes of rest, standing blood pressure measurements were obtained using an automatic, calibrated sphygmomanometer (GE Healthcare). Radial artery flow-mediated dilatation (FMD) was then measured as previously described (Dragoni et al. 2005; Gori et al. 2007; Gori et al. 2005). Briefly, study subjects rested in a semi-supine position for 20 minutes, 
after which, end-diastolic, ECG-gated, longitudinal, B-mode images of the artery 10-15 cm below the antecubital fossa were digitally acquired and stored for off-line analysis. Arterial diameter was recorded continuously for 1 minute before cuff inflation (resting diameter), during the period of distal cuff inflation (4'30") and for another 4'30" after wrist cuff deflation. Semiautomatic custom-designed software that allowed for image acquisition was employed to calculate the arterial diameter from the trailing edge to the leading edge of the interface between the intima and blood. Subsequently, a pneumatic cuff placed at the level of the brachial artery and inflated to $250 \mathrm{~mm} \mathrm{Hg}$ for 15 minutes was used to induce forearm ischemia. At the end of this period of ischemia, 15 minutes of reperfusion were allowed before FMD was measured again. This model, which causes a marked impairment in endothelium-dependent vasodilation while leaving endothelium-independent vasodilation intact (Kharbanda et al. 2001), has been recently employed in multiple studies of IR injury and preconditioning (Dragoni et al. 2005; Gori et al. 2007; Gori et al. 2005). The repeatability and reproducibility of FMD as assessed in our laboratory have been described previously (Gori et al. 2008). Venous blood samples were taken at the end of the visits for the analysis of sex steroid hormones (estradiol, progesterone, testosterone) and to assess lipid levels.

Subjects returned to the laboratory to have their second visit coincide with either the LF or EF phase of the menstrual cycle (i.e. the opposite of the first visit), and the procedures described above were repeated. 


\section{Statistical Analysis}

Data are presented as mean \pm SEM. Within-phase and between-phase differences, as well as the interaction of IR and follicular phase were studied with a 2-way ANOVA for repeated measures. Post-hoc comparisons were performed using the Bonferroni correction. The relationship between estradiol concentration and the level of preconditioning (i.e. change in FMD after IR) was determined with a logarithmic regression analysis, using the equation: $Y=b 0+b 1 * \ln (X)$ (where $b 0$ is the $\mathrm{y}$-intercept and $b 1$ is the regression coefficient of $x$ (i.e. slope of the regression line)). A value of $P<0.05$ was set as the threshold for significance. SAS 9.1.3 (SAS Institute Inc. Cary, NC) was used for all statistical analyses.

\section{Results}

\section{Sex Steroid Analysis}

Results are shown in Figure 1. Serum estradiol levels during the EF and LF phases of the menstrual cycle were $148.4 \pm 19.8 \mathrm{pmol} / \mathrm{L}$ and $825.7 \pm 85.8 \mathrm{pmol} / \mathrm{L}$, respectively $(P<0.001)$, representing a five-fold increase in estradiol during the LF phase. There were no differences in serum progesterone and testosterone concentrations between the two phases (progesterone; EF: $2.2 \pm 0.9 \mathrm{nmol} / \mathrm{L}, \mathrm{LF}: 2.4 \pm 0.7 \mathrm{nmol} / \mathrm{L}, P=\mathrm{NS}$; testosterone; EF: $2.1 \pm 0.1 \mathrm{nmol} / \mathrm{L}, \mathrm{LF}: 2.5 \pm 0.2$ $\mathrm{nmol} / \mathrm{L}, P=\mathrm{NS})$.

\section{Baseline Parameters}

There were no significant differences between groups (i.e. EF vs. LF phase) in either baseline blood flow or reactive hyperemia (percent increase in blood flow after wrist cuff deflation) before and after IR. Further, FMD was similar before IR (Table 2 and Figure 2). Baseline 
diameters before IR and after IR were not significantly different between the phases of the menstrual cycle. Systolic blood pressure (SBP) and mean arterial pressure (MAP) were significantly lower during the LF phase (SBP; EF: $109 \pm 1 \mathrm{mmHg}$; LF: $104 \pm 2 \mathrm{mmHg} ; P=0.02$; MAP; EF: $82 \pm 1 \mathrm{mmHg}$; LF: $79 \pm 2 ; P=0.04)$.

\section{Effect of Ischemia and Reperfusion}

IR significantly reduced FMD during the EF phase (Figure 2, before IR: $7.1 \pm 1.0 \%$; after IR: 3.6 $\pm 1.0 \%, P=0.01)$. In contrast, FMD was not significantly different following IR during the LF phase (Figure 2: before IR; 7.2\% $\pm 1.0 \%$; after IR: $7.0 \% \pm 0.8 \% ; P=\mathrm{NS}$ vs. LF before IR). The FMD responses following IR during the LF phase were significantly higher compared to those observed after IR during the EF phase $(P=0.03$ for the interaction of IR and phase of the menstrual cycle; $P=0.02$ versus post-IR in the EF phase (Figure 2)).

\section{Relationship Between Estradiol Concentration and Level of Preconditioning}

A logarithmic regression analysis was performed to determine whether there was a statistically significant relationship between estradiol concentrations and the level of preconditioning (i.e. the change in FMD after IR) (Figure 3). The data were described by the following equation: $Y=$ $13.282-1.97 * \ln (X)$. A significant inverse relationship was found between estradiol concentration and change in FMD after IR $\left(r=0.59, r^{2}=0.36, P<0.01\right)$.

\section{Discussion}

The present study was designed to determine whether there are differences in forearm endothelium-dependent vasomotor responses following IR in the EF vs. the LF phase of the 
menstrual cycle. Our findings indicate that the LF of the cycle is associated with essentially complete preservation of endothelial function in response to forearm IR injury. We hypothesize that this protective effect results from the dramatic differences in circulating estradiol levels observed during the 2 phases of the menstrual cycle. The regression analysis demonstrates that there is a significant inverse relationship between estradiol concentration and the change in FMD after IR, such that as estradiol levels increase, there is less likelihood that endothelial function will be blunted after injury. The current observations are the first demonstration of a protective effect of cyclical increases of estradiol in the setting of IR injury in humans and are consistent with animal studies in which endogenous or exogenous estrogens were shown to reduce infarct size, increase ventricular functional recovery, and reduce inflammation following myocardial IR injury (Murphy and Steenbergen 2007). These beneficial effects are thought to be mediated by non-genomic, receptor-activated signaling cascades such as the phosphatidyl-inositol-3-kinase pathway as well as through nuclear receptor-mediated induced expression of protective genes (eg. endothelial nitric oxide synthase (NOS) and neuronal NOS) (Murphy and Steenbergen 2007).

The molecular pathways through which estradiol may have provided the protective effects in our study have been explored in mechanistic experiments performed in animal models of IR. Das et al. demonstrated that pre-treatment of intact rabbits with $17-\beta$-estradiol prior to IR resulted in significantly reduced infarct size and incidence of life-threatening arrhythmias compared to saline and vehicle control groups. Importantly, these protective effects were abolished with the co-administration of the mitochondrial ATP-dependent potassium channel $\left(\mathrm{mK}_{\mathrm{ATP}}\right)$ blocker 5hydroxydecanoate, indicating a potential mechanistic role for the $\mathrm{mK}_{\mathrm{ATP}}$ in the protective effects 
of 17- $\beta$-estradiol (Das and Sarkar 2006). Booth et al showed that rabbits treated with estradiol had a significantly reduced infarct size following IR injury compared to control animals, an effect that was eliminated in the presence of the COX-2 inhibitor nimesulide (Booth et al. 2008). Finally, in an eloquent study performed by Lagranha and colleagues, isolated female rabbit hearts that exhibited a protected phenotype in the setting of IR compared to males and ovariectomized females were shown to have increased phosphorylation and activity of the mitochondrial enzyme aldehyde dehydrogenase-2 (ALDH2) (Lagranha et al. 2010), an enzyme that has recently been shown to be an important mediator of preconditioning (Chen et al. 2008). Interestingly, the ALDH-2 inhibitor wortmannin abolished the protective phenotype in female but not in male rabbits that had been treated with exogenous estrogen (Lagranha et al. 2010).

Several studies in young, healthy, women have shown that endothelium-mediated responses at baseline as measured by brachial artery FMD are greater during the surge of estradiol that occurs in the LF phase of the menstrual cycle compared to the EF phase (Hashimoto et al. 1995) (Williams et al. 2001). A recent finding described the ability of nine-month treatment with a second-generation oral contraceptive (consisting of $30 \mu \mathrm{g}$ ethinyl estradiol and $150 \mu \mathrm{g}$ levonorgestrel) to significantly increase brachial FMD values in a cohort of young, female endurance athletes with amenorrhea, a condition that is associated with endothelial dysfunction (Rickenlund et al. 2005). Unlike these findings, our data do not indicate a significant difference in baseline FMD between the EF and LF phases of the menstrual cycle. It is possible that differences in brachial versus radial artery physiology, which have been shown to be governed by different processes (Thijssen et al. 2008) may have played a role in this discrepancy. Furthermore, our cohort of healthy, normally-menstruating subjects cannot be directly compared 
to women with amenorrhea, whose long duration of estrogen deficiency as well as other metabolic and hormonal abnormalities have been suggested to have complex effects on vascular structure and function (O'Donnell et al. 2007).

The fact that the present data were acquired in healthy volunteers and in the conduit circulation of the forearm, a vasculature different from the coronary circulation, is acknowledged as a limitation. However, the model employed in the present study has been previously shown to provide reliable and relevant information of the effect of IR injury on the vasculature (Kharbanda et al. 2001). Study of the endothelium has particular relevance, since this tissue is the most sensitive to IR, and since damage of the (macro- and microvascular) endothelium can prevent effective reperfusion in spite of timely intervention. For these reasons, we believe that the study of potential therapies and treatment regimens aimed at protecting the endothelium in the setting of IR should be considered clinically relevant. Following IR the baseline radial diameter of the radial artery was smaller than that observed post IR during the early follicular phase. Although this difference was not statistically significant it could have had an impact on the differences observed in FMD responses between the early and late follicular phases. Finally, in the current study we used noninvasive approaches to address endothelial function and did not test vascular responses to endothelium-dependent vasodilators such as acetylcholine. However, it should be noted that this forearm model of IR has previously been shown to blunt the responses to endothelium-dependent vasodilators just as it blunts the responses to FMD (Kharbanda et al, 2001). 
In conclusion, the present study demonstrates, for the first time in humans, that the surge in estradiol that occurs in the LF phase of the menstrual cycle attenuates the endothelial dysfunction associated with a forearm model of IR in healthy pre-menopausal women. These results support animal studies in which estrogens have been shown to provide protective effects in the setting of IR.

\section{Acknowledgements}

The authors thank the staff of the John H. Daniels Cardiac Research Centre and the Mecklinger and Posluns Cardiac Catheterization Research Laboratory of the Mount Sinai Hospital, Toronto.

\section{Funding Sources}

This study was funded by a grant from the Heart and Stroke Foundation of Ontario. The study was supported by a grant-in-aid from the Heart and Stroke Foundation of Ontario.

\section{Disclosures.}

None. 


\section{Reference List}

Abela, C.B., and Homer-Vanniasinkham, S. 2003. Clinical implications of ischaemia-reperfusion injury. Pathophysiology, 9(4): 229-240.

Bolli, R. 2000. The late phase of preconditioning. Circ. Res. 87(11): 972-983.

Booth, E.A., Flint, R.R., Lucas, K.L., Knittel, A.K., and Lucchesi, B.R. 2008. Estrogen protects the heart from ischemia-reperfusion injury via COX-2-derived PGI2. J. Cardiovasc. Pharmacol. 52(3): 228-235.

Chen, C.H., Budas, G.R., Churchill, E.N., Disatnik, M.H., Hurley, T.D., and Mochly-Rosen, D. 2008. Activation of aldehyde dehydrogenase- 2 reduces ischemic damage to the heart. Science, 321(5895): 1493-1495.

Das, B., and Sarkar, C. 2006. Similarities between ischemic preconditioning and 17beta-estradiol mediated cardiomyocyte KATP channel activation leading to cardioprotective and antiarrhythmic effects during ischemia/reperfusion in the intact rabbit heart. J. Cardiovasc. Pharmacol. 47(2): 277-286.

Dragoni, S., Gori, T., Di, S.G., Sicuro, S., Forconi, S., and Parker, J.D. 2005. Folic Acid does not limit endothelial dysfunction induced by ischemia and reperfusion: a human study. J.

Cardiovasc. Pharmacol. 46(4): 494-497.

Dragoni, S., Gori, T., Lisi, M., Di Stolfo, G., Pautz, A., Kleinert, H., and Parker, J.D. 2007. Pentaerythrityl tetranitrate and nitroglycerin, but not isosorbide mononitrate, prevent endothelial dysfunction induced by ischemia and reperfusion. Arterioscler. Thromb. Vasc. Biol. 27(9): 19551959.

Dubey, R.K., Imthurn, B., Barton, M., and Jackson, E.K. 2005. Vascular consequences of menopause and hormone therapy: importance of timing of treatment and type of estrogen. Cardiovasc. Res. 66(2): 295-306.

Gori, T., Di Stolfo, G., Sicuro, S., Dragoni, S., Lisi, M., Forconi, S., and Parker, J.D. 2007. Nitroglycerin protects the endothelium from ischaemia and reperfusion: human mechanistic insight. Br. J. Clin. Pharmacol. 64(2): 145-150.

Gori, T., Dragoni, S., Lisi, M., Di Stolfo, G., Sonnati, S., Fineschi, M., and Parker, J.D. 2008. Conduit artery constriction mediated by low flow a novel noninvasive method for the assessment of vascular function. J. Am. Coll. Cardiol. 51(20): 1953-1958.

Gori, T., Sicuro, S., Dragoni, S., Donati, G., Forconi, S., and Parker, J.D. 2005. Sildenafil prevents endothelial dysfunction induced by ischemia and reperfusion via opening of adenosine triphosphate-sensitive potassium channels: a human in vivo study. Circulation, 111(6): 742-746.

Guermandi, E., Vegetti, W., Bianchi, M.M., Uglietti, A., Ragni, G., and Crosignani, P. 2001. Reliability of ovulation tests in infertile women. Obstet. Gynecol. 97(1): 92-96. 
Hashimoto, M., Akishita, M., Eto, M., Ishikawa, M., Kozaki, K., Toba, K., Sagara, Y., Taketani, Y., Orimo, H., and Ouchi, Y. 1995. Modulation of endothelium-dependent flow-mediated dilatation of the brachial artery by sex and menstrual cycle. Circulation, 92(12): 3431-3435.

Kharbanda, R.K., Peters, M., Walton, B., Kattenhorn, M., Mullen, M., Klein, N., Vallance, P., Deanfield, J., and MacAllister, R. 2001. Ischemic preconditioning prevents endothelial injury and systemic neutrophil activation during ischemia-reperfusion in humans in vivo. Circulation, 103(12): 1624-1630.

Lagranha, C.J., Deschamps, A., Aponte, A., Steenbergen, C., and Murphy, E. 2010. Sex differences in the phosphorylation of mitochondrial proteins result in reduced production of reactive oxygen species and cardioprotection in females. Circ. Res. 106(11): 1681-1691.

Liuni, A., Luca, M.C., Gori, T., and Parker, J.D. 2010. Rosuvastatin prevents conduit artery endothelial dysfunction induced by ischemia and reperfusion by a cyclooxygenase-2-dependent mechanism. J. Am. Coll. Cardiol. 55(10): 1002-1006.

Murphy, E., and Steenbergen, C. 2007. Cardioprotection in females: a role for nitric oxide and altered gene expression. Heart Fail. Rev. 12(3-4): 293-300.

O'Donnell, E., Harvey, P.J., Goodman, J.M., and De Souza, M.J. 2007. Long-term estrogen deficiency lowers regional blood flow, resting systolic blood pressure, and heart rate in exercising premenopausal women. Am. J. Physiol. 292(5): E1401-1409.

Rickenlund, A., Eriksson, M.J., Schenck-Gustafsson, K., and Hirschberg, A.L. 2005. Oral contraceptives improve endothelial function in amenorrheic athletes. J. Clin. Endocrinol. Metab. 90(6): 3162-3167.

Thijssen, D.H., Dawson, E.A., Black, M.A., Hopman, M.T., Cable, N.T., and Green, D.J. 2008. Heterogeneity in conduit artery function in humans: impact of arterial size. Am. J. Physiol.

295(5): H1927-1934.

Williams, M.R., Westerman, R.A., Kingwell, B.A., Paige, J., Blombery, P.A., Sudhir, K., and Komesaroff, P.A. 2001. Variations in endothelial function and arterial compliance during the menstrual cycle. J. Clin. Endocrinol. Metab. 86(11): 5389-5395.

Zhu, B., Sun, Y., Sievers, R.E., Browne, A.E., Pulukurthy, S., Sudhir, K., Lee, R.J., Chou, T.M., Chatterjee, K., and Parmley, W.W. 2000. Comparative effects of pretreatment with captopril and losartan on cardiovascular protection in a rat model of ischemia-reperfusion. J. Am. Coll. Cardiol. 35(3): 787-795. 
Table 1: Participant Characteristics

\begin{tabular}{ll}
\hline Mean age (years) & $22.9 \pm 3.0$ \\
Caucasian (\%) & $80 \%$ \\
South Asian (\%) & $10 \%$ \\
Black (\%) & $10 \%$ \\
Mean cycle length (days) & $26.8 \pm 1.3$ \\
\% with LH surge detected in $\mathbf{2 / 2}$ monitored cycles & $90 \%$ \\
\% with LH surge detected in 1/2 monitored cycles & $10 \%$ \\
\hline
\end{tabular}

Data are presented as mean \pm standard deviation. 
Table 2: Arterial diameter and Blood Flow Data

\section{BEFORE IR}

DIAMETER (mm) Baseline

Early Follicular

Late Follicular

$1.88 \pm 0.01$

$1.93 \pm 0.01$

\section{Maximum after cuff release}

$2.02 \pm 0.01$

$2.07 \pm 0.01$

$1.91 \pm 0.03$

$1.81 \pm 0.01$

Baseline

AFTER IR

BLOOD FLOW (ml/min)

$\begin{array}{lllll}\text { Early Follicular } & 4.1 \pm 1.2 & 88.2 \pm 18.2 & 2.9 \pm 0.7 & 79.6 \pm 14.9 \\ \text { Late Follicular } & 7.7 \pm 2.5 & 84.5 \pm 13.1 & 3.7 \pm 0.9 & 79.6 \pm 11.7\end{array}$

Data are presented as mean \pm standard error. Radial artery diameter and blood flow before wrist cuff occlusion (baseline) and during reactive hyperemia (maximum values between 30 seconds and 4 minutes after wrist cuff deflation) are presented.

$* P<0.01$ for $\%$ change from baseline compared to before IR 


\section{Figure Legends}

Figure 1. Change in serum concentrations of estradiol, progesterone and testosterone between the EF and LF phases of the menstrual cycle. Concentrations of estradiol were significantly higher during the LF phase compared to the EF phase whereas there were no significant differences in concentrations of progesterone and testosterone between the two phases of the menstrual cycle.

$* P<0.001$ compared to EF; EF, early follicular; LF, late follicular.

Figure 2. Percent increase in radial artery diameter during reactive hyperemia (FMD) before and after IR. While FMD in the EF phase was significantly attenuated after IR, FMD was not significantly different compared to before IR in the LF phase. FMD, flow-mediated dilation; IR, ischemia and reperfusion; EF, early follicular; LF, late follicular.

Figure 3. Logarithmic regression demonstrating the relationship between estradiol concentrations and the level of preconditioning (i.e. the change in FMD after IR). A significant inverse relationship was shown between estradiol concentrations and the change in FMD after IR. 
Figure 1

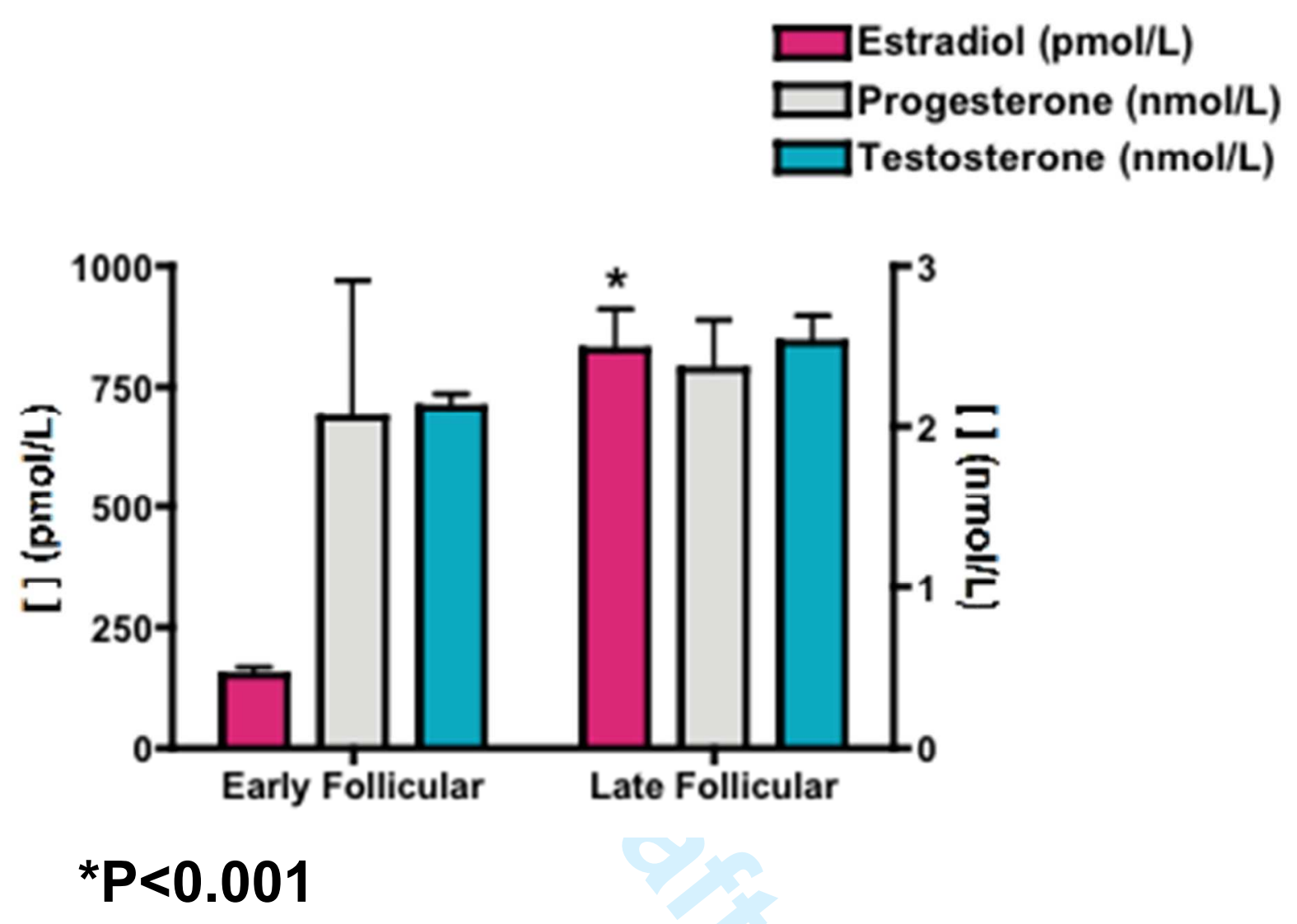


Figure 2

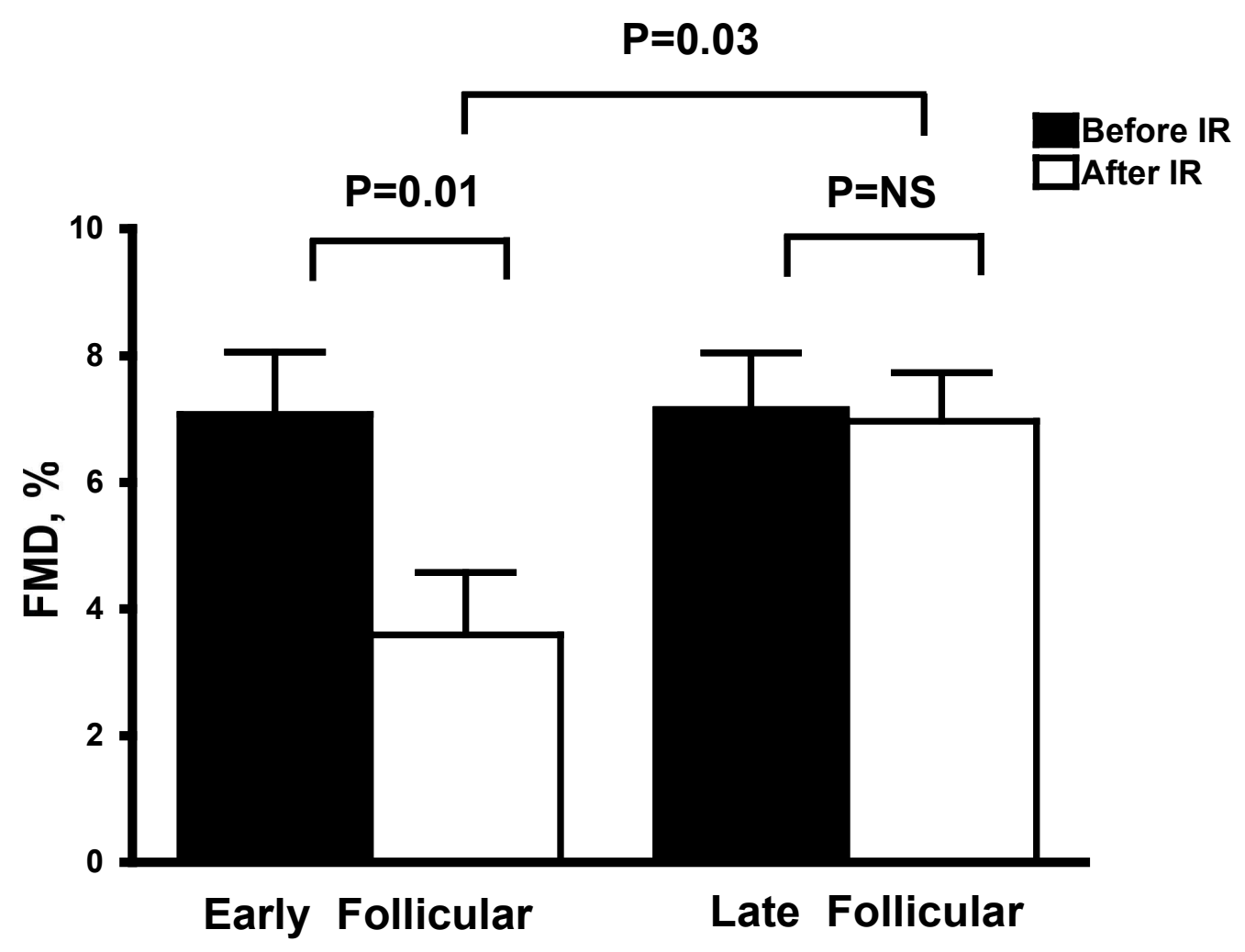


Figure 3

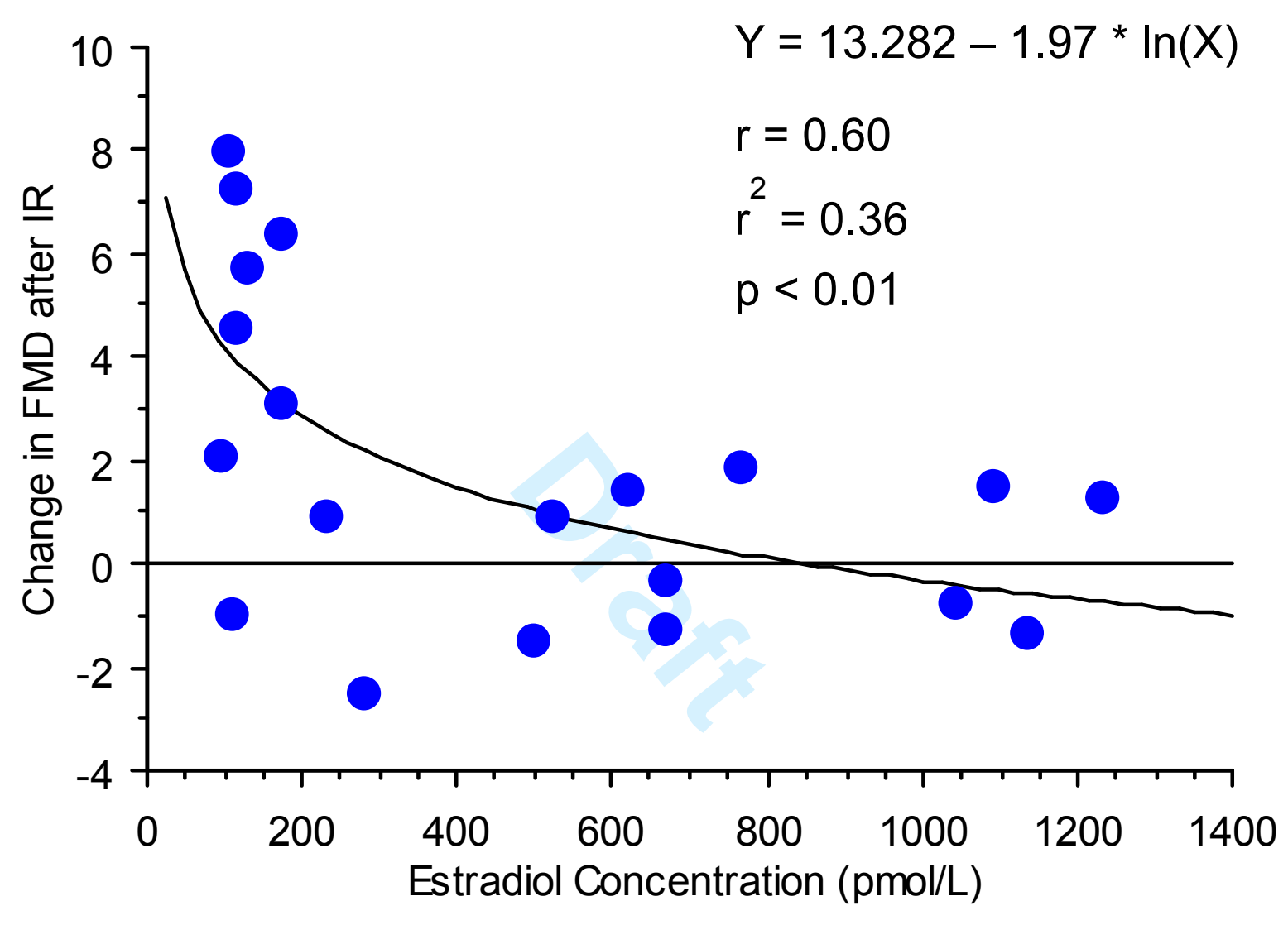

